\title{
Editorial
}

\section{Alcohol use during pregnancy and fetal alcohol spectrum disorder in Canada: who, what, where?}

\author{
Jocelynn L. Cook, PhD $(1,2,3)$
}

\section{Understanding fetal alcohol spectrum disorder}

The estimated prevalence of fetal alcohol spectrum disorder (FASD) in Canada is greater than that of autism, cerebral palsy and Down syndrome combined, and the incremental cost per case of FASD over a person's lifespan is estimated at $\$ 1.1$ million. ${ }^{1}$

FASD is a lifelong disability that impacts the brain and body of individuals prenatally exposed to alcohol. Individuals frequently have challenges in their daily living and need support with motor skills, physical health, learning, memory, emotional regulation and social skills., ${ }^{2,3}$ Emerging evidence suggests that FASD is also associated with chronic disease and mental health disorders and adverse societal experiences. ${ }^{4,5}$ When undiagnosed and unsupported, individuals with FASD are more likely to experience substance use challenges, mental health conditions, involvement with the criminal justice and child welfare systems, emotional and physical abuse, trauma and disrupted housing. ${ }^{6}$

FASD occurs within a web of increased risk and vulnerabilities. All populations that use alcohol are at risk for FASD, yet we still do not understand the prevalence of alcohol consumption during pregnancy, or FASD, in Canada.

\section{Alcohol use during preǵnancy}

That alcohol is a neurobehavioural teratogen is neither contentious nor debatable. Yet, despite years of public messaging about the harms of alcohol during pregnancy, and millions of dollars spent on prevention campaigns, the number of women who consume alcohol during pregnancy has not decreased. ${ }^{?}$

So what's going on?

We know that the lives of women who drink alcohol during pregnancy are complex. We know that women consume alcohol during pregnancy for a number of reasons, that the social determinants of health play an important role $^{8}$ and that stigma impacts reporting alcohol use and accessing interventions. ${ }^{9}$ And sometimes women don't know that they are pregnant.

Of note, the newest iteration of the guideline on screening and counselling for alcohol consumption during pregnancy, published in September 2020 by the Society of Obstetricians and Gynaecologists of Canada, ${ }^{10}$ explicitly states that all pregnant women should be questioned about alcohol use. If a pattern of use is established, this information should be documented in the infant's medical record after delivery. These recommendations attempt to incorporate alcohol screening and conversations about alcohol use into routine practice, and to note exposure in both the mother's and the baby's charts. Health care providers play a critical role in capturing this information and getting it into provincial data systems, and normalizing questions about alcohol use and creating a safe environment to have meaningful conversations with patient-clients.

\section{Surveillance}

Canada's surveillance data related to alcohol and pregnancy are scarce. There are a few published reports, and many people have worked very hard to try to capture routine information about alcohol and pregnancy with complete ascertainment, using retrospective and prospective approaches and over time and across jurisdictions. There have been national surveys, including the now defunct Maternity Experiences Survey and the still operational Canadian Community Health Survey, that have provided a snapshot of use. While these provide information about the amount of alcohol reported to have been consumed, we do not have information about the reasons for use, contexts for use, challenges/barriers to accessing supports and services, or patterns of consumption and at which points during pregnancy. And the information that is available is based on self-report data with values too small, in many cases, to be useful for drawing general conclusions.

Provincial databases and registries sometimes capture information related to alcohol use during pregnancy, but they depend upon information about alcohol use being captured in the data source (i.e. patient charts). While this approach could capture information about alcohol use during pregnancy relatively thoroughly, the details are often not available and the datasets tend to be incomplete. Alcohol use is almost exclusively self-reported, and with the complexity of stigma, coupled with the fact that screening for alcohol use during pregnancy is not a standard part of prenatal/antenatal forms, the data tend to not be reliable. There have been attempts to develop a standardized national perinatal record that includes information about prenatal exposures, but efforts appear to have stalled.

1. Chief Scientific Officer, The Society of Obstetricians and Gynaecologists of Canada, Ottawa, Ontario, Canada

2. National Database Lead, Canada FASD Research Network, Vancouver, British Columbia, Canada

3. Adjunct Professor, Department of Obstetrics and Gynecology, University of Ottawa, Ottawa, Ontario, Canada

Correspondence: Jocelynn L. Cook, Chief Scientific Officer, The Society of Obstetricians and Gynaecologists of Canada, 2781 Lancaster Road, Ottawa, ON K1B 1A7; Email: jcook@sogc.com 
Canada also has no set of indicators or systematic and routine data collection related to the prevalence of FASD. Again, the research community has done a lot of excellent work attempted to glean prevalence data from different studies in different populations in Canada. We have reports from rural communities, ${ }^{11}$ urban schools, ${ }^{12}$ child welfare systems ${ }^{13}$ and justice systems. ${ }^{14}$ In 2019, the Canadian Health Survey on Children and Youth attempted to gather data about FASD. ${ }^{15}$ These studies depend on the results of an FASD diagnostic assessment, and Canada's access to diagnostic services falls very short of demand. ${ }^{16}$ This is a critical gap: without early identification of prenatal alcohol exposure captured by our data systems, we lose the opportunity for early monitoring and anticipatory guidance for supports and services. And the opportunity to maximize potential is tragically missed.

Capturing reliable information about amounts of alcohol and patterns of use/prenatal exposure in provincial data systems would go a long way toward understanding prevalence, demographic and regional trends as well as comorbidities. We would also be able to pinpoint time periods where alcohol consumption, as well as comorbidities, seem to change-the past year of the COVID-19 pandemic is a perfect example-in order to anticipate the need for changes in prevention and support programs. This type of data would also help us to better understand the effectiveness of prevention and harm reduction efforts in real time-or at least much faster than the years it takes to report on national surveys.

\section{The National FASD Database}

The National FASD Database is another national project that has been implemented to capture thorough information about FASD, risk factors and outcomes. From 2010 to 2020, the Public Health Agency of Canada provided support for the development and implementation of the National FASD Database. NeuroDevNet/ Kids Brain Health contributed to the support from 2013 to 2019, and the Canada FASD Research Network is now the sole funder. The database was developed in an attempt to capture information about FASD and its prevalence across all Canada's diagnostic clinics.
Presently, we have over 3500 records, and have learned about the environments of individuals who were exposed to alcohol prenatally, their health and behaviours and the challenges to changing the way we think about preventing FASD and supporting women who use alcohol during pregnancy.

Despite limited diagnostic capacity in Canada, with the full participation of clinics across the country we can ascertain prevalence of FASD using a system such as the national database.

\section{Summary and recommendation for funding and support}

Alcohol use during pregnancy, and FASD, is complex, and we need to understand the magnitude of the issue as well as the situations surrounding it. Provincial data systems are poised to capture information about alcohol use, including during pregnancy, as well as patterns and amounts consumed. Integrating these questions into the routine practice of health care providers is critical. Standardizing perinatal forms would also be a helpful contribution.

Canada has come a long way, and in many ways we are leaders in the field of FASD research. But our fragmented health system with jurisdictional differences in data collection and barriers to data sharing make it difficult to report nationally on the prevalence of alcohol use during pregnancy and FASD. Further developing the National FASD Database as part of the pan-Canadian surveillance infrastructure, with buy-in and participation from all jurisdictions, is an effective way to capture and report on accurate national data.

\section{References}

1. Thanh NX, Jonsson E. Costs of fetal alcohol spectrum disorder in the Canadian criminal justice system. J Popul Ther Clin Pharmacol. 2015; 22(1):e125-31.

2. McLachlan K, Flannigan K, Temple V, Unsworth K, Cook JL. Difficulties in daily living experienced by adolescents, transition-aged youth, and adults with fetal alcohol spectrum disorder. Alcohol Clin Exp Res. 2020;44(8): 1609-24. https://doi.org/10.1111/acer .14385
3. Skorka K, McBryde C, Copley J, Meredith PJ, Reid N. Experiences of children with fetal alcohol spectrum disorder and their families: a critical review. Alcohol Clin Exp Res. 2020; 44(6):1175-88. https://doi.org/10.1111 /acer. 14335

4. Lebel CA, Gibbard WB, Tortorelli C, et al. Prenatal Exposure And Child brain and mental Health (PEACH) study: protocol for a cohort study of children and youth with prenatal alcohol exposure. BMJ Open. 2021; 11(5):e051660. https://doi.org/10.1136 /bmjopen-2021-051660

5. Flannigan K, Kapasi A, Pei J, Murdoch I, Andrew G, Rasmussen C. Characterizing adverse childhood experiences among children and adolescents with prenatal alcohol exposure and fetal alcohol spectrum disorder. Child Abuse Negl. 2021;112:104888. https:// doi.org/10.1016/j.chiabu.2020.104888

6. Popova S, Temple V, Dozet D, O’Hanlon G, Toews C, Rehm J. Health, social and legal outcomes of individuals with diagnosed or at risk for fetal alcohol spectrum disorder: Canadian example. Drug Alcohol Depend. 2021; 219:108487. https://doi.org/10.1016/j .drugalcdep.2020.108487

7. Burd L. Drinking at the end of pregnancy: why don't we see it? Pediatr Res. 2020;88(2):142. https://doi.org /10.1038/s41390-020-0846-1

8. Terplan M. Alcohol-exposed pregnancy outcomes explained by social determinants of health. BJOG. 2015;122(13): 1739. https://doi.org/10.1111/1471 $-0528.13216$

9. Escañuela Sánchez T, Matvienko-Sikar K, Linehan L, O’Donoghue K, Byrne M, Meaney S. Facilitators and barriers to substance-free pregnancies in highincome countries: a meta-synthesis of qualitative research. Women Birth. 2021:S1871-5192(21)00076-7. https:// doi.org/10.1016/j.wombi.2021.04.010

10. Graves L, Carson G, Poole N, et al. Guideline No. 405: Screening and counselling for alcohol consumption during pregnancy. J Obstet Gynaecol Can. 2020;42(9):1158-73.e1. https:// doi.org/10.1016/j.jogc.2020.03.002 
11. Robinson GC, Conry JL, Conry RF. Clinical profile and prevalence of fetal alcohol syndrome in an isolated community in British Columbia. CMAJ. 1987;137(3):203-7.

12. Popova S, Lange S, Poznyak V, et al. Population-based prevalence of fetal alcohol spectrum disorder in Canada. BMC Public Health. 2019;19(1):845. https://doi.org/10.1186/s12889 $-019-7213-3$

13. Popova S, Lange S, Burd L, Rehm J. Canadian children and youth in care: the cost of fetal alcohol spectrum disorder. Child Youth Care Forum. 2014;43(1):83-96. https://doi.org/10 .1007/s10566-013-9226-X

14. McLachlan K, McNeil A, Pei J, Brain U, Andrew G, Oberlander TF. Prevalence and characteristics of adults with fetal alcohol spectrum disorder in corrections: a Canadian case ascertainment study. BMC Public Health. 2019;19(1):43. https://doi.org/10.1186 /s12889-018-6292-x

15. Statistics Canada. Canadian Health Survey on Children and Youth (CHSCY) [Internet]. Ottawa (ON): Statistics Canada; [modified 2019 Feb 11; cited 2021 May 17]. Available at: https:// www23.statcan.gc.ca/imdb/p2SV .pl Function $=$ getSurvey $\&$ SDDS $=5233$

16. Clarren SK, Lutke J, Sherbuck M. The Canadian guidelines and the interdisciplinary clinical capacity of Canada to diagnose fetal alcohol spectrum disorder. J Popul Ther Clin Pharmacol. 2011;18(3):e494-9. 